Louisiana State University

LSU Digital Commons

Faculty Publications

Department of Physics \& Astronomy

$5-29-2020$

\title{
Spontaneous Creation of Circularly Polarized Photons in Chiral Astrophysical Systems
}

\author{
Adrian Del Rio \\ Instituto Superior Técnico \\ Nicolas Sanchis-Gual \\ Instituto Superior Técnico \\ Vassilios Mewes \\ Oak Ridge National Laboratory \\ Ivan Agullo \\ Louisiana State University \\ José A. Font \\ Universitat de València
}

See next page for additional authors

Follow this and additional works at: https://digitalcommons.Isu.edu/physics_astronomy_pubs

\section{Recommended Citation}

Del Rio, A., Sanchis-Gual, N., Mewes, V., Agullo, I., Font, J., \& Navarro-Salas, J. (2020). Spontaneous Creation of Circularly Polarized Photons in Chiral Astrophysical Systems. Physical Review Letters, 124 (21) https://doi.org/10.1103/PhysRevLett.124.211301

This Article is brought to you for free and open access by the Department of Physics \& Astronomy at LSU Digital Commons. It has been accepted for inclusion in Faculty Publications by an authorized administrator of LSU Digital Commons. For more information, please contact ir@lsu.edu. 
Authors

Adrian Del Rio, Nicolas Sanchis-Gual, Vassilios Mewes, Ivan Agullo, José A. Font, and Jose Navarro-Salas 


\title{
Spontaneous Creation of Circularly Polarized Photons in Chiral Astrophysical Systems
}

\author{
Adrian del Rio, ${ }^{1}$ Nicolas Sanchis-Gualø, ${ }^{1}$ Vassilios Mewes $\odot,{ }^{2,3,4}$ Ivan Agullo, ${ }^{5}$ \\ José A. Font, ${ }^{6,7}$ and Jose Navarro-Salas ${ }^{8}$ \\ ${ }^{1}$ Centro de Astrofísica e Gravitação-CENTRA, Instituto Superior Técnico, Universidade de Lisboa, \\ Avenida Rovisco Pais 1, 1049-001, Lisboa, Portugal \\ ${ }^{2}$ National Center for Computational Sciences, Oak Ridge National Laboratory, \\ P.O. Box 2008, Oak Ridge, Tennessee 37831-6164, USA \\ ${ }^{3}$ Physics Division, Oak Ridge National Laboratory, P.O. Box 2008, Oak Ridge, Tennessee 37831-6354, USA \\ ${ }^{4}$ Center for Computational Relativity and Gravitation, and School of Mathematical Sciences, Rochester Institute of Technology, \\ 85 Lomb Memorial Drive, Rochester, New York 14623, USA \\ ${ }^{5}$ Department of Physics and Astronomy, Louisiana State University, Baton Rouge, Louisiana 70803-4001, USA \\ ${ }^{6}$ Departamento de Astronomía y Astrofísica, Universitat de València, Doctor Moliner 50, 46100 Burjassot (València), Spain \\ ${ }^{7}$ Observatori Astronòmic, Universitat de València, Catedrático José Beltrán 2, 46980 Paterna (València), Spain \\ ${ }^{8}$ Departamento de Física Teórica and IFIC, Universitat de València-CSIC. \\ Doctor Moliner 50, 46100 Burjassot (València), Spain
}

(Received 12 February 2020; revised manuscript received 23 March 2020; accepted 4 May 2020; published 27 May 2020)

\begin{abstract}
This work establishes a relation between chiral anomalies in curved spacetimes and the radiative content of the gravitational field. In particular, we show that a flux of circularly polarized gravitational waves triggers the spontaneous creation of photons with net circular polarization from the quantum vacuum. Using waveform catalogs, we identify precessing binary black holes as astrophysical configurations that emit such gravitational radiation and then solve the fully nonlinear Einstein's equations with numerical relativity to evaluate the net effect. The quantum amplitude for a merger is comparable to the Hawking emission rate of the final black hole and small to be directly observed. However, the implications for the inspiral of binary neutron stars could be more prominent, as argued on symmetry grounds.
\end{abstract}

DOI: 10.1103/PhysRevLett.124.211301

Introduction.-Maxwell equations without charges and currents are invariant under duality rotations of the electromagnetic field, $F_{a b} \rightarrow F_{a b} \cos \theta+{ }^{\star} F_{a b} \sin \theta$. This continuous transformation was shown to be a physical symmetry of the source-free Maxwell action, $S=$ $-\frac{1}{4} \int_{M} d^{4} x \sqrt{-g} F_{a b} F^{a b}(x)$, in a general curved spacetime background $\left(M, g_{a b}\right)$ [1]. By Noether's theorem, this symmetry leads to a conserved current, $\nabla_{a} j_{D}^{a}=0$. Its associated Noether charge $Q_{D}$ physically accounts for the difference between right- and left-handed circularly polarized components [2] - the usual Stokes V parameter-as one could expect, since duality rotations are just chiral transformations for the electromagnetic field. Thus, this symmetry guarantees that the circular polarization state of classical electromagnetic radiation is a constant of motion, even if the field propagates in an arbitrary gravitational background.

However, the quantum theory offers a more interesting scenario [3]. In the presence of gravity, quantum fluctuations of the electromagnetic field can spoil the classical conservation law as $\left\langle 0\left|\nabla_{a} j_{D}^{a}\right| 0\right\rangle=\left(-1 / 96 \pi^{2}\right) R_{a b c d}{ }^{\star} R^{a b c d} \neq 0$, where $|0\rangle$ is any vacuum state and $R_{a b c}{ }^{d}$ is the Riemann tensor of the spacetime. This is understood as the spin-1 generalization of the Fermionic chiral anomaly [4]. The goal of this paper is to explore the physical consequences of this nonconservation law and to identify astrophysical systems where the new effect could be relevant.

Chiral flux of photons and the importance of gravitational dynamics.-The anomalous current indicates that the Noether charge is no longer conserved in time and that its change is determined by the geometry of the background. More precisely, for asymptotically Minkowski spacetimes [5], where we can compare the values of the Noether charge at past and future null infinities, $\mathcal{J}^{ \pm}$(where the gravitational interaction vanishes and preferred notions of quantum vacua exist), one finds

$$
\left\langle Q_{D}\left(\mathcal{J}^{+}\right)\right\rangle-\left\langle Q_{D}\left(\mathcal{J}^{-}\right)\right\rangle=\frac{-1}{96 \pi^{2}} \int_{M} d^{4} x \sqrt{-g} R_{a b c d}{ }^{\star} R^{a b c d} .
$$

If the electromagnetic field is initially in vacuum, $\left\langle Q_{D}\left(\mathcal{J}^{-}\right)\right\rangle=0$, a nonzero value at late times, $\left\langle Q_{D}\left(\mathcal{J}^{+}\right)\right\rangle \neq 0$, indicates that a flux of photons with net circular polarization arrives at $\mathcal{J}^{+}$. This happens only if the right-hand side of Eq. (1) is different from zero. This quantity is called the Chern-Pontryagin, and it measures the degree of spacetime chirality. It is dimensionless, as is the 
number operator in the left-hand side. The helicity of photons can be measured by direct detections at $\mathcal{J}^{+}$ through polarimetry observations; hence a good understanding of the right-hand side is fundamental to making precise predictions.

While a nonzero value of the Chern-Pontryagin is usually associated with the presence of certain gravitational instantons [6], these are solutions of the Euclidean Einstein's equations only, whose interpretation as quantum-tunneling mediators between different topologies [7] relies on quantum gravity considerations. Our interest is in solutions of a more direct physical interpretation. We aim to identify Lorentzian geometries satisfying Einstein's equations having a nonzero Chern-Pontryagin and then evaluate Eq. (1) for such classical gravitational fields. This is a nontrivial task, since physically realistic exact solutions to Einstein's equations provide a null contribution to Eq. (1). For instance, black hole $(\mathrm{BH})$ solutions of the KerrNewman family yield a vanishing value after integrating over all angles. This is because the Chern-Pontryagin is a pseudoscalar (flips sign under improper rotations), while the underlying Kerr-Newman metric is even under mirror reflections with respect to the plane perpendicular to the symmetry axis. We must consider spacetimes with no mirror symmetries. Uniqueness theorems in general relativity [8] assert that any regular, asymptotically flat, stationary vacuum solution in four dimensions is a member of the above family. Since we are interested in asymptotically flatness - to have a good notion of photons and helicity at asymptotic times - the above considerations suggest that relevant scenarios will only come up if we give up stationarity.

The lack of stationarity makes the analysis highly nontrivial and would require, in general, numerical methods. Below we provide a physical characterization of spacetimes having a nonzero Chern-Pontryagin, finding in turn a deep interplay between handedness of photons and circularly polarized gravitational waves (GWs). This is a new and purely gravitational effect, which is in sharp contrast to the instanton solutions reported in the literature (which are only topological, not geometrical).

Flux of circularly polarized GWs.-By the Chern-Weyl theorem from the theory of characteristic classes [9], given $R_{a b c d}, R_{a b c d}^{\prime}$, the curvature tensors of any two connection 1 -forms $\omega_{a}, \omega_{a}^{\prime}$ on $M$, the difference $d^{4} x \sqrt{-g} R_{a b c d}{ }^{\star} R^{a b c d}-$ $d^{4} x \sqrt{-g^{\prime}} R_{a b c d}^{\prime}{ }^{\star} R^{\prime a b c d}=d T\left(\omega, \omega^{\prime}\right)$ is exact and determined by the so-called transgression term $T\left(\omega, \omega^{\prime}\right)$, whose explicit expression can be found in [6]. Taking $\omega^{\prime}$ as any flat connection of Minkowski spacetime [10], we have $R_{a b c d}^{\prime}{ }^{\star} R^{\prime a b c d}=0$ [12]. Consider now $M_{R}=$ $\{p \in M / r(p) \leq R\} \subset M$, with $r$ a radial coordinate centered at the sources and $R$ a sufficiently large number so that all gravitational sources are well inside $M_{R}$. We can use the Stokes theorem to write the volume integral of $M_{R}$ as the integral over the boundary,

$$
\int_{M_{R}} d^{4} x \sqrt{-g} R_{a b c d^{\star}} R^{a b c d}=\int_{\partial M_{R}} T\left(\omega, \omega^{\prime}\right) .
$$

We now take the limit $M_{R} \rightarrow M$. If we assume no incoming GWs and isolated astrophysical sources, nontrivial contributions are expected only from future null infinity. Following Bondi-Sachs [14], we foliate the asymptotic region of $M$ around $\mathcal{J}^{+}$by outgoing null hypersurfaces $u=$ const (physically representing retarded time), each one generated by null geodesics of affine parameter $r$ and take the limit $r \rightarrow \infty$ along these geodesics to reach $\mathcal{J}^{+}$. Using then the Newman-Penrose spin-coefficient formalism [15] and the asymptotic properties developed in [11], one can find [16]

$\frac{-1}{96 \pi^{2}} \int_{M} d^{4} x \sqrt{-g} R_{a b c d^{\star}} R^{a b c d}=\int_{-\infty}^{\infty} d u f(u) \equiv \Delta Q_{\mathcal{J}^{+}}$,

where [17]

$$
f(u)=\frac{1}{72 \pi^{2}} \int_{-\infty}^{u} d u^{\prime} \sum_{\ell m} \operatorname{Im}\left[\Psi_{4, \ell m}^{0}(u) \bar{\Psi}_{4, \ell m}^{0}\left(u^{\prime}\right)\right] .
$$

In this formula, $\Psi_{4}^{0}(u, \theta, \phi)=\lim _{r \rightarrow \infty} r \Psi_{4}(u, r, \theta, \phi)$ is the leading order coefficient of the Weyl scalar $\Psi_{4}$ [15], and we have expanded the angular part in spherical harmonics of spin weight -2 . Although this expression may look complicated, its physical interpretation is remarkably simple. Recall that the usual gravitational wave (GW) polarization modes $h_{+}, h_{\times}$are in correspondence with $\Psi_{4}^{0}$ by $\partial_{u}^{2} h_{+}=\operatorname{Re} \Psi_{4}^{0}, \partial_{u}^{2} h_{\times}=\operatorname{Im} \Psi_{4}^{0}$. If we expand $h_{\times}$and $h_{+}$ in Fourier modes, then Eq. (3) is equal to

$$
\begin{aligned}
& \int_{0}^{\infty} \frac{d \omega \omega^{2}}{72 \pi^{2}} \sum_{\ell m}\left[\left|h_{+}^{\ell m}(\omega)+i h_{\times}^{\ell m}(\omega)\right|^{2}\right. \\
& \left.-\left|h_{+}^{\ell m}(\omega)-i h_{\times}^{\ell m}(\omega)\right|^{2}\right],
\end{aligned}
$$

which measures the difference in intensity between right and left circularly polarized GWs reaching future null infinity. This is the Stokes V parameter of GWs. Thus, from Eq. (1) we conclude that the emission of chiral gravitational radiation by astrophysical systems implies the spontaneous creation of photons with net helicity. The more right- or left-handed GWs the spacetime contains, the more left or right-handed photons will be excited from the quantum vacuum.

In the presence of a $\mathrm{BH}, \mathcal{J}^{+}$alone is not a Cauchy hypersurface, and one needs to add the contribution from the future event horizon $H$ to Eqs (3) and (4), i.e., the information that does not escape to infinity but rather falls into the singularity. This contribution can be computed in a similar manner, but the final expression is complicated and 
does not have a simple interpretation. However, it can be checked that for an isolated horizon [18] the contribution is zero, and from this one infers that a flux of chiral gravitational perturbations across $H$ is needed in order to contribute to the Chern-Pontryagin.

Identification of chiral astrophysical systems using $G W$ data.-A nonvanishing value of Eq. (4) can be used as a sufficient condition to identify chiral systems. Mergers of binary black holes (BBHs) and binary neutron stars (BNSs), such as those detected by advanced LIGO and Virgo [19], are promising candidates. Since numerical relativity simulations of compact binary mergers are computationally expensive, we first calculate Eqs. (3) and (4) using GW data from freely available catalogs [20-23] to identify suitable spin and mass configurations. Binary black hole $(\mathrm{BBH})$ catalogs are extensive, and this allows us to efficiently cover a large range of parameter space. Binary neutron star (BNS) simulations, however, are scarce and only some conclusions can be drawn at present. With the most suitable BBH candidates, we perform numerical simulations in the following section to calculate the integral Eq. (1).

Results from illustrative models of $\mathrm{BBH}$ mergers are shown in Table I. We find no clear dependence with mass ratio, $\mathrm{BH}$ spins, merger time, or GW luminosity. Interestingly, only precessing systems produce non-negligible values. These occur when the spins of the black holes (BHs) are misaligned with the binary's orbital angular momentum. Mergers of spin-aligned objects yield values compatible with zero because positive and negative $m$ modes are found to cancel each other up to roundoff error for all $\ell$ in Eq. (4). This result can be understood as follows. Consider the functional $F\left[g_{a b}\right]=\int_{\Sigma} d^{3} x \sqrt{-g} R_{a b c d}{ }^{*} R^{a b c d}$. As a pseudoscalar, it changes sign under a reflection $I$ (improper rotation) and remains invariant under a proper rotation $R$, namely $(R \circ I)\left(F\left[g_{a b}\right]\right)=F\left[(R \circ I) g_{a b}\right]=-F\left[g_{a b}\right]$. Suppose now a binary system with metric $g_{a b}$, in which the two spins remain always aligned. By taking a mirror reflection with respect to the separation plane, we can always return to the same configuration $g_{a b}$ after a suitable rotation. In other words, $F\left[(R \circ I) g_{a b}\right]=F\left[g_{a b}\right]$. This leads to $F\left[g_{a b}\right]=0$, which explains the observed result. This argument fails if the two spins are misaligned and justifies the observed nonzero values in Table I for precessing binaries.

Table I also shows that the values obtained for some available BNS mergers are 3 to 4 orders of magnitude smaller than those for precessing $\mathrm{BBH}$ mergers. This is because, despite the fact that the orbital plane may precess, the individual neutron star spins are actually very low and aligned. The fact that the values are not compatible with zero (unlike the $\mathrm{BBH}$ case) must be due to the complex multipolar structure of NS. Nontrivial higher order multipole moments, not determined by the mass and spin, encode (axial-type) deformations of the Kerr gravitational field that break the perfect symmetry under mirror reflections. In this respect, notice that even a merger of nonrotating NS leads to a nonzero value.

$B B H$ simulations. - We next perform numerical simulations of $\mathrm{BBH}$ mergers to gauge the contribution of the $\mathrm{BH}$ horizon to the Chern-Pontryagin. We solve Einstein's equations numerically for the spacetime metric to calculate Eq. (1). The 4D integral can be easily split in the usual $3+1$ formalism employed in numerical relativity [24] by noticing that $R_{a b c d^{\star}} R^{a b c d}=16 E_{a b} B^{a b}$, where $E_{a b}$ and $B^{a b}$ are the electric and magnetic parts of the Weyl tensor, respectively. These are purely spatial tensors, so given two spacelike Cauchy hypersurfaces $\Sigma_{1}, \Sigma_{2} \subset \mathbb{R}^{3}$ we have:

TABLE I. Values of $\Delta Q_{\mathcal{J}^{+}}$for a representative sample of BBHs (obtained from the RIT catalog [20-22]) and some BNS mergers (obtained in [23], with the SLy equation of state). Types NS, A, and P correspond to nonspinning, aligned, and precessing binaries, respectively. The spins of the two initial objects, $\vec{\chi}_{1}$ and $\vec{\chi}_{2}$, are shown in Cartesian coordinates, and $q=m_{1} / m_{2}$ indicates the mass ratio. The GW peak luminosity for all BBH models fluctuates around $\sim 10^{56} \mathrm{erg} \mathrm{s}^{-1}$. BBH-0062 corresponds to LIGO event GW150914.

\begin{tabular}{|c|c|c|c|c|c|c|c|c|c|}
\hline Model & & $q$ & $\chi_{1 x}$ & $\chi_{1 y}$ & $\chi_{1 z}$ & $\chi_{2 x}$ & $\chi_{2 y}$ & $\chi_{2 z}$ & $72 \pi^{2} \Delta Q_{\mathcal{J}^{+}}$ \\
\hline ВBH-0001 & NS & 1.00 & 0 & 0 & 0 & 0 & 0 & 0 & $-4.0 \times 10^{-14}$ \\
\hline BBH-0038 & $P$ & 1.00 & 0.77 & 0 & -0.20 & 0 & 0 & 0.20 & 0.17 \\
\hline ВBH-0062 & $A$ & 0.82 & 0 & 0 & -0.44 & 0 & 0 & 0.33 & $9.4 \times 10^{-13}$ \\
\hline BBH-0068 & $A$ & 1.00 & 0 & 0 & -0.80 & 0 & 0 & -0.80 & $-6.5 \times 10^{-13}$ \\
\hline ВBH-0105 & $A$ & 0.33 & 0 & 0 & 0.80 & 0 & 0 & 0 & $2.9 \times 10^{-11}$ \\
\hline BBH-0130 & $P$ & 0.75 & 0 & 0 & -0.80 & 0.53 & 0 & 0.60 & 0.14 \\
\hline ВBH-0137 & $P$ & 0.50 & -0.35 & 0 & 0.35 & 0.35 & 0 & 0.35 & 0.12 \\
\hline BBH-0272 & $P$ & 0.67 & 0 & 0 & 0 & 0 & 0.69 & 0.40 & -0.22 \\
\hline $\operatorname{BNS}(o o)$ & NS & 0.82 & 0 & 0 & 0 & 0 & 0 & 0 & $6.2 \times 10^{-5}$ \\
\hline $\operatorname{BNS}(u u)$ & $A$ & 0.82 & 0 & 0 & 0.08 & 0 & 0 & 0.09 & $-1.3 \times 10^{-4}$ \\
\hline $\operatorname{BNS}(d d)$ & $A$ & 0.82 & 0 & 0 & -0.08 & 0 & 0 & -0.09 & $-5.1 \times 10^{-5}$ \\
\hline $\operatorname{BNS}(n n)$ & $P$ & 0.82 & 0.08 & 0.08 & 0.08 & 0.09 & 0.09 & 0.09 & $2.0 \times 10^{-4}$ \\
\hline $\mathrm{BNS}(s s)$ & $P$ & 0.82 & -0.08 & -0.08 & -0.08 & -0.09 & -0.09 & -0.09 & $-7.4 \times 10^{-5}$ \\
\hline
\end{tabular}


TABLE II. Net photon helicity computed from Eq. (5) with numerical relativity simulations of orbital mergers and head-on collisions of BHs. The contribution from GW, Eq. (3), is given for comparison.

\begin{tabular}{lccccc}
\hline \hline Type & $q$ & $D / 2 M$ & $a_{i} / m_{i}$ & $\Delta Q_{\mathcal{J}^{+}}$ & $\left\langle Q_{D}\left(t_{2}\right)\right\rangle-\left\langle Q_{D}\left(t_{1}\right)\right\rangle$ \\
\hline Orbital NS & 1.0 & 2.5 & 0.00 & $2.0 \times 10^{-15}$ & $3.4 \times 10^{-11}$ \\
Orbital $A$ & 1.0 & 2.1 & 0.62 & $1.2 \times 10^{-14}$ & $6.4 \times 10^{-11}$ \\
Orbital $P$ & 1.0 & 4.5 & 0.63 & $1.2 \times 10^{-4}$ & 0.08 \\
Orbital $P$ & 1.0 & 8.1 & 0.63 & $1.3 \times 10^{-4}$ & 0.02 \\
Head-on & 1.0 & 8.3 & 0.63 & $5.1 \times 10^{-6}$ & 0.07 \\
\hline \hline
\end{tabular}

$\left\langle Q_{D}\left(t_{2}\right)\right\rangle-\left\langle Q_{D}\left(t_{1}\right)\right\rangle=\frac{-1}{6 \pi^{2}} \int_{t_{1}}^{t_{2}} d t \int_{\Sigma_{t}} d \Sigma \sqrt{-g} E_{a b} B^{a b}$.

We compute this by modifying the ANTENNA thorn [25] and perform simulations using the Einstein Toolkit [26], using the McLachlan thorn [27] for the spacetime evolution. To simulate quasicircular BBH mergers, we take the component masses and initial linear momentum from [28], in particular those corresponding to BHs initially separated by $D / 2=\{4.5 M, 8.1 M\}$, in units of the ADM mass $M$. The initial data are calculated using the TwoPunctures thorn [29]. In both cases we consider BHs with spins tilted $45^{\circ}$ with respect to the orbital plane and $90^{\circ}$ with respect to each other: $(\leftarrow, 0, \uparrow),(\rightarrow, 0, \uparrow)$. For comparison, we also evolve head-on collisions of $\mathrm{BHs}$ with antialigned spins: $(\rightarrow, 0,0),(\leftarrow, 0,0)$. The results are shown in Table II.

To gain further intuition, Fig. 1 shows snapshots of the evolution of $E_{a b} B^{a b}(x, y)$ from the inspiral-mergerringdown phases of two Kerr BHs with the same spin magnitude and $D / 2=8.1 M$. The time evolution of $Q(t):=$ $\int_{\Sigma_{t}} d^{3} x \sqrt{-g} E_{a b} B^{a b}$ is shown in the red curve of Fig. 2. Despite the high nonlinearities, using symmetry considerations as above we can qualitatively understand its behavior in the last orbit of the inspiral. At $t \sim 137 M$, the two spins are pointing inwards the orbit (see Fig. 1), corresponding to a maximal configuration, thus explaining the negative peak found. At $156 M$, the two objects have evolved one quarter of an orbit, and their spins have
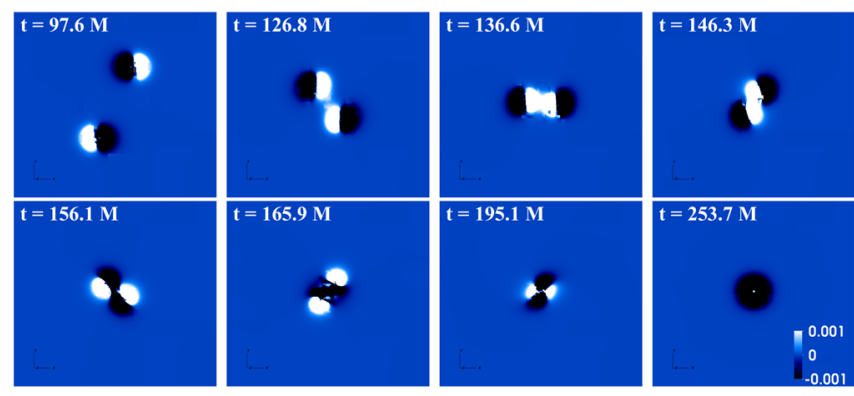

FIG. 1. Time evolution of $E_{a b} B^{a b}$ in the $z=0$ coordinate plane for a $\mathrm{BBH}$ merger with parameters $q=1, D / 2=8.1 M$, and $a_{1,2} / m_{1,2}=0.63$. acquired a relative orientation that is even under mirror reflections with respect to the separation plane, yielding a vanishing value. When the system has evolved another quarter of an orbit, at time $166 \mathrm{M}$, the two spins point outwards the orbit; this is the mirror-reflected initial configuration and explains the observed positive maximum peak. After another quarter of an orbit (at 180M), the two objects again reach a configuration that is even under mirror reflections, producing another zero value. Returning to the initial configuration after a full cycle (at 190M), the spatial integral attains the maximum negative peak observed, right before the merger. The amplitude of the oscillations grows because the relative distance between the BHs decreases significantly in the last orbits. After merger, the value of Eq. (5) decays to zero, as corresponds to a final Kerr BH.

The inset of Fig. 2 shows the evolution of $Q(t)$ for orbital mergers of two Schwarzschild and two Kerr BHs with spins aligned with the orbital plane. In both cases, the result is zero up to roundoff error for any time, as expected from symmetry considerations. This confirms the conclusions drawn in the previous section using waveform catalogs.

Discussion.-The close relation between chiral anomalies and circular polarization established in Eq. (1) and Eqs. (3) and (4) implies that a source of chiral GW induces the excitation from the quantum vacuum of a flux of photons with net circular polarization, $\left\langle Q_{D}\left(\mathcal{J}^{+}\right)\right\rangle \neq 0$. This is not accounted for by gravitational instantons. Furthermore, for any numerical simulation in relativistic astrophysics, Eqs. (3) and (4) can be used to identify the emergence of this quantum effect by simply relying on the resulting gravitational waveforms. In fact, it could be evaluated from LIGO/Virgo detections directly. This work, hence, builds a bridge between numerical relativity,

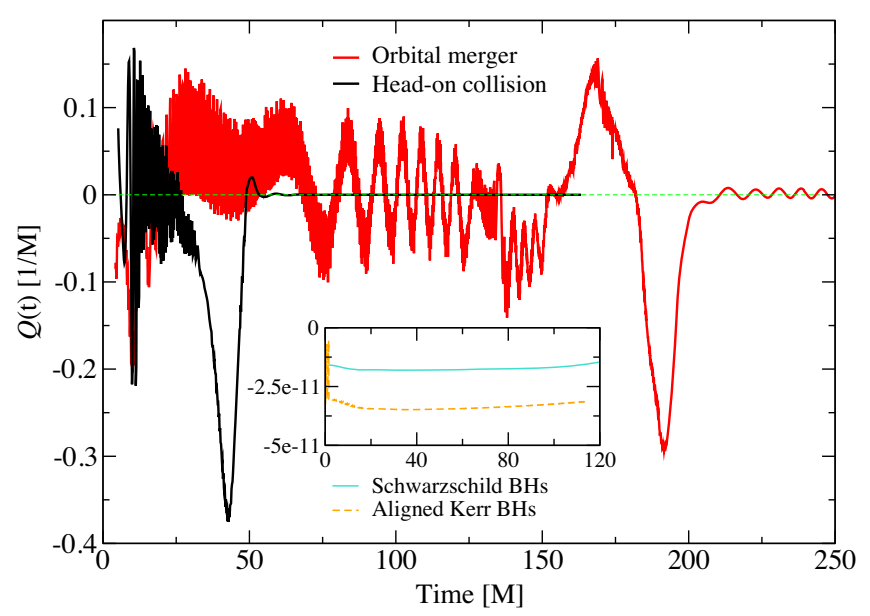

FIG. 2. Time evolution of the spatial integral in Eq. (5) for an orbital merger (red curve) and head-on collision (black curve) of misaligned Kerr BHs. The inset corresponds to orbital mergers of both Schwarzschild and aligned Kerr BHs, compatible with zero for all time. 
GW observations, and quantum field theory in curved spacetimes.

Although the Hawking effect for stationary BHs predicts an angular-dependent helicity distribution [30], the net contribution when integrated over all angles is zero, in agreement with the vanishing of Eq. (1) for a Kerr metric. We report here the possibility of generating net helicity in spacetimes that have no mirror symmetries, as for example during collisions of BHs with misaligned spins. In this case, we find a peak at the time of merger with value $5 \times 10^{-3} / M$, which is comparable to the total Hawking emission rate of photons by the final $\mathrm{BH}$ configuration, $10^{-3} / M$ [31]. Note in passing that both effects, although intrinsically different, scale similarly.

The results obtained for astrophysical BBH coalescences are small to be observed directly, mainly due to the quantum gravitational origin of the effect. However, its emergence can act as a "seed" that, together with possible classical mechanisms of amplification, might lead to more observable predictions. Without such mechanisms, the amplitude of this quantum effect seems of observational relevance only if there exists an accumulative process over long periods of time. One example could be the inspiral phase of compact binaries, which last millions of years. The contribution from BBHs with adiabatic orbital precession is expected to be negligible because the spin configurations at different times along the orbit are related by mirror reflection, and consequently they cancel each other when integrating in time; only highly (orbital and/or spin) precessing inspirals could be important. On the other hand, BNS systems, which have a richer multipolar structure and can be endowed with strong magnetic fields, can avoid the cancellations in time and lead to predictions of interest. Let us suppose an idealized BNS system in which each star has a misaligned rotation axis and magnetic field axis. If the rotation of the components is synchronized with the orbital motion, each magnetic field will spin around at the same rate. The spatial configuration of the four axial vectors will thus remain approximately invariant during the evolution. Equations (3) and (4) yield an estimate of $\Delta Q_{\mathcal{J}^{+}} \approx 10^{3}$ in 1-2 years of observation, by assuming that the contribution of Eq. (4) is in the same ballpark as for the precessing BBH cases. Future developments of BNS numerical simulations involving higher (and misaligned) spin values and/or magnetic fields will help to quantify this effect in realistic scenarios and to estimate its chances of being measured in multimessenger astronomy observations of BNS mergers such as GW170817 [32].

Our analysis finds applications in other contexts as well. For instance, the physical effect described in this paper can be straightforwardly extended to fermions, since they also have a chiral anomaly dictated by the Chern-Pontryagin [33]. Therefore, a source of circularly polarized GWs (or electromagnetic waves $[4,17]$ ) can also trigger the creation of chiral fermions. For neutrinos, this is equivalent to creation of leptonic asymmetry. On the other hand, our results could also be applied to analyze the stability of magnetic fields in neutron stars [34]. A nonvanishing magnetic helicity is needed to avoid the decay of magnetic fields, but its origin remains unclear. Using [35], it can be deduced that a nonzero Chern-Pontryagin produces a nonzero magnetic helicity by quantum fluctuations, and, according to our results, this can be achieved in a core collapse supernova emitting circularly polarized GWs [36].

As a by-product of this work, we notice that GW circular polarization can be a useful measurement from LIGO/Virgo observations to extract information about $\mathrm{BBH}$ mergers since, as shown above, its detection would be a clear indication for the existence of precession, something that frequently has only a weak imprint on the observable waveform [37].

We are grateful to T. Dietrich, V. Chaurasia and collaborators for providing waveform data from their BNS simulations. We thank A. Ashtekar, E. Bianchi, M. Campanelli, M. Campiglia, V. Cardoso, M. Casals, F. Duque, G. Faye, S. Gimeno-Soler, C. Herdeiro, A. Torres-Forne, J. Zanelli, and Y. Zlochower for useful comments. This work also benefited from discussions during the GR22/Amaldi13 conference in Valencia. A. D. R. acknowledges financial support by the European Union's H2020 ERC Consolidator Grant "Matter and strong-field gravity: New frontiers in Einstein's theory" No. MaGRaTh-646597, and funds from the GWverse COST Action CA16104, "Black holes, gravitational waves, and fundamental physics"; N. S. G. by the Fundação para a Ciência e a Tecnologia (FCT) projects No. PTDC/FISOUT/28407/2017 and No. UIDB/00099/2020; J. A. F. by the Spanish Agencia Estatal de Investigación (Grant No. PGC2018-095984-B-I00), the Generalitat Valenciana (PROMETEO/2019/071) and the European Union's Horizon 2020 RISE programme (H2020-MSCA-RISE-2017 Grant No. FunFiCO-777740); I. A. by the NSF CAREER Grant No. PHY-1552603, and the Hearne Institute for Theoretical Physics of Louisiana State University; J. N. S. by the Ministerio de Economia y Competitividad grants No. FIS2017-91161-EXP and No. FIS2017-84440-C2-1P; and V. M. by NSF Grants No. OAC-1550436, No. AST1516150, No. PHY-1607520, No. PHY-1305730, No. PHY1707946, No. PHY-1726215, funds from AYA2015-66899C2-1-P, and Rochester Institute of Technology for the FGWA SIRA initiative, the Exascale Computing Project (17-SC-20SC), a collaborative effort of the U.S. Department of Energy (DOE) Office of Science and the National Nuclear Security Administration. Work at Oak Ridge National Laboratory is supported under contract No. DE-AC05-00OR22725 with the U.S. Department of Energy.

[1] S. Deser and C. Teitelboim, Phys. Rev. D 13, 1592 (1976). [2] M. G. Calkin, Am. J. Phys. 33, 958 (1965). 
[3] I. Agullo, A. del Rio, and J. Navarro-Salas, Phys. Rev. Lett. 118, 111301 (2017); Int. J. Mod. Phys. D 26, 1742001 (2017); Phys. Rev. D 98, 125001 (2018); Symmetry 10, 763 (2018).

[4] S. L. Adler, Phys. Rev. 177, 2426 (1969); J. S. Bell and R. Jackiw, Nuovo Cimento A 60, 47 (1969).

[5] A. Ashtekar, in Surveys in Differential Geometry, edited by L. Bieri and S. T. Yau (International Press, Boston, 2015), p. 99.

[6] T. Eguchi, P. B. Gilkey, and A. J. Hanson, Phys. Rep. 66, 213 (1980).

[7] G. W. Gibbons and S. W. Hawking, Phys. Lett. 78B, 430 (1978).

[8] P. T. Chrusciel, J. L. Costa, and M. Heusler, Living Rev. Relativity 15, 7 (2012).

[9] M. Nakahara, Geometry, Topology and Physics, Graduate Student Series in Physics (Hilger, Bristol, UK, 1990).

[10] The choice $\omega^{\prime}=0$ recovers the well-known Chern-Simons 3 -form for $T$. We keep $\omega^{\prime}$ general to use the results of Ref. [11], based on coordinate systems with $\omega^{\prime} \neq 0$.

[11] E. T. Newman and R. Penrose, J. Math. Phys. (N.Y.) 3, 566 (1962); S. Chandrasekhar, The Mathematical Theory of Black Holes (Clarendon, Oxford, UK, 1985).

[12] By taking this auxiliary metric, we implicitly assume that our manifold is $M \subset \mathbb{R}^{4}$, since other $M$ may not admit a flat connection globally. The topology in the problem is thus trivial, and this is supported by the topological censorship theorem in general relativity [13].

[13] J. L. Friedman, K. Schleich, and D. M. Witt, Phys. Rev. Lett. 71, 1486 (1993).

[14] H. Bondi, M. G. J. van der Burg, and A. W. K. Metzner, Proc. R. Soc. A 269, 21 (1962); R. Sachs, Phys. Rev. 128, 2851 (1962).

[15] E. T. Newman and T. W. Unti, J. Math. Phys. (N.Y.) 3, 891 (1962).

[16] A. del Rio et al. (to be published).

[17] An analogous expression holds for the electromagnetic Chern-Pontryagin [16].

[18] A. Ashtekar, C. Beetle, and J. Lewandowski, Classical Quantum Gravity 19, 1195 (2002).

[19] B. P. Abbott et al. (LIGO Scientific and Virgo Collaborations), Phys. Rev. X 9, 031040 (2019).
[20] G. Lovelace, C. O. Lousto, J. Healy et al., Classical Quantum Gravity 33, 244002 (2016).

[21] J. Healy, C. O. Lousto, Y. Zlochower, and M. Campanelli, Classical Quantum Gravity 34, 224001 (2017).

[22] J. Healy, C. O. Lousto, J. Lange, R. O’Shaughnessy, Y. Zlochower, and M. Campanelli, Phys. Rev. D 100, 024021 (2019).

[23] T. Dietrich, S. Bernuzzi, B. Brugmann, M. Ujevic, and W. Tichy, Phys. Rev. D 97, 064002 (2018).

[24] M. Alcubierre, Introduction to 3+1 Numerical Relativity (Oxford Science Publication, Oxford, 2008).

[25] M. Campanelli, B. J. Kelly, and C. O. Lousto, Phys. Rev. D 73, 064005 (2006); J. G. Baker, M. Campanelli, and C. O. Lousto, Phys. Rev. D 65, 044001 (2002).

[26] EinsteinToolkit, Einstein Toolkit: Open software for relativistic astrophysics, http://einsteintoolkit.org/.F. Löffler, Classical Quantum Gravity 29, 115001 (2012).

[27] D. Brown, P. Diener, O. Sarbach, E. Schnetter, and M. Tiglio, Phys. Rev. D 79, 044023 (2009); C. Reisswig, C. D. Ott, U. Sperhake, and E. Schnetter, Phys. Rev. D 83, 064008 (2011).

[28] W. Tichy and B. Brügmann, Phys. Rev. D 69, 024006 (2004).

[29] M. Ansorg, B. Brügmann, and W. Tichy, Phys. Rev. D 70, 064011 (2004).

[30] A. Vilenkin, Phys. Rev. Lett. 41, 1575 (1978); Phys. Rev. D 20, 1807 (1979); D. A. Leahy and W. G. Unruh, Phys. Rev. D 19, 3509 (1979).

[31] M. Casals (private communication); D. N. Page, Phys. Rev. D 14, 3260 (1976).

[32] B. P. Abbott et al. (LIGO Scientific and Virgo Collaborations), Phys. Rev. Lett. 119, 161101 (2017); Astrophys. J. 848, L12 (2017); 848, L13 (2017).

[33] T. Kimura, Prog. Theor. Phys. 42, 1191 (1969).

[34] H. C. Spruit, AIP Conf. Proc. 983, 391 (2008).

[35] A. D. Dolgov, I. B. Khriplovich, A. I. Vainshtein, and V. I. Zakharov, Nucl. Phys. B315, 138 (1989).

[36] K. Hayama, T. Kuroda, K. Nakamura, and S. Yamada, Phys. Rev. Lett. 116, 151102 (2016); K. Hayama, T. Kuroda, K. Kotake, and T. Takiwaki, Mon. Not. R. Astron. Soc. 477, L96 (2018).

[37] S. Fairhurst, R. Green, C. Hoy, M. Hannam, and A. Muir, arXiv:1908.05707. 